medical therapy. Patients with IGG4-HBD respond well to medical therapy and Rituximab can be used effectively in their management.

\section{PWE-16 INJECTING NETWORK STRUCTURE DETERMINES THE MOST EFFICIENT STRATEGY FOR HEPATITIS C ELIMINATION}

${ }^{1}$ Ryan Buchanan*, ${ }^{1}$ Chloe Brown, ${ }^{2}$ Martin Siegele, ${ }^{3}$ Rachel Sacks-Davis, ${ }^{4}$ Mark Wright, ${ }^{1}$ Salim I Khakoo, 'Julie Parkes. 'Faculty of Medicine, University Of Southampton, Southampton, UK; ${ }^{2}$ School of Mathematical and Physical Sciences, University of Sussex, Brighton, UK; ${ }^{3}$ Burnett Institute, Melbourne, Australia; ${ }^{4}$ Hepatology Department, University Hospital Southampton, Southampton, UK

10.1136/gutjpl-2021-BSG.206

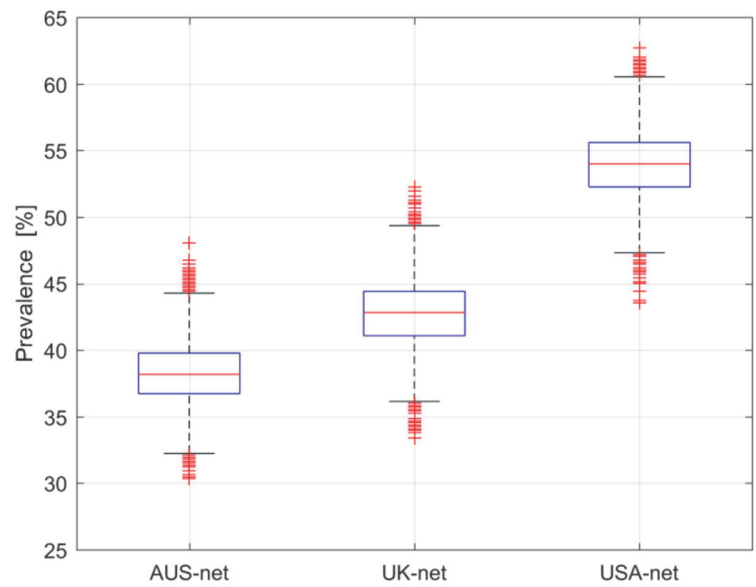

(a)

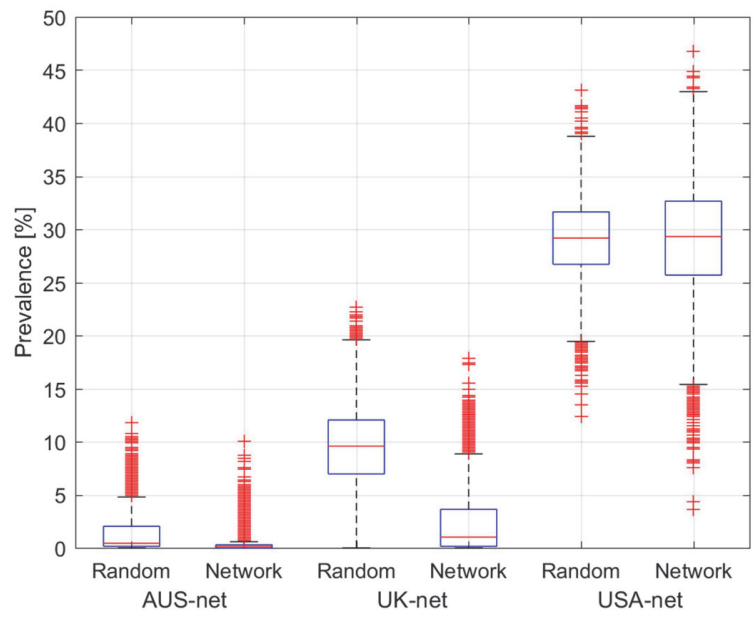

(b)

Abstract PWE-16 Figure 1 Box plots showing prevalence of HCV at 5 years, for each network structure from a baseline prevalence of 41.7\%: (a) without treatment; (b) treating 6 PWID per month by each of random and degree-based targeted ('network') strategies. The ends of the boxes are the upper and lower quartiles, a horizontal line inside each box marks the median value and the whiskers extend to extreme values at most $1.5 x$ the inter-quartile range. Outliers beyond this range are indicated by crosses
Introduction Transmission of Hepatitis C (HCV) continues via sharing of injection equipment between people who inject drugs (PWID). Network-based modelling studies have produced conflicting results as to whether random treatment is preferable to targeting treatment at PWID with multiple partners. We hypothesise that differences in network structure produce this heterogeneity, and aim to test how changing network structure affects HCV transmission and treatment effects. Methods We create three different dynamic injecting network structures connecting 689 PWID (UK-net, AUS-net and USAnet) based on published empirical data. HCV within the networks is transmitted via a susceptable-infected-susceptable model. At 5 years we compare prevalence of $\mathrm{HCV}$ in the three networks in three scenarios: 1) with no treatment, 2) with randomly targeted treatment and 3) with treatment targeted at PWID with the most injecting partnerships.

Results Median HCV prevalence at 5 years without treatment differed significantly between the three networks (UK-net 42.8\%; AUS-net 38.2\%, $p<0.0001$; USA-net 54.0\%, $p<$ 0.0001). In the treatment scenarios UK-net showed a clear benefit of targeted treatment (median 5 -year prevalence 1.0\% vs. $9.6 \%$ vs. $\mathrm{p}<0.0001)$, AUS-net showed a smaller benefit $(0.15 \%$ vs. $0.44 \%, p<0.0001)$ and USA-net showed no significant difference $(29.3 \%$ vs. $29.2 \%$ random, $p=0.0681)$. In sensitivity analyses, targeted treatment was optimised in low prevalence, moderate treatment coverage conditions whereas random treatment was optimised in low treatment coverage, high baseline prevalence conditions.

Conclusions Network structure determines the transmission rate of HCV in PWID and the most efficient treatment strategy to achieve elimination. In real-world injecting network structures, the benefit of targeting HCV treatment at individuals with multiple injecting partnerships may have been underestimated. Therefore, focussing additional treatment resources at actively injecting PWID who are less engaged with harm reduction may be worthwhile.

\section{PWE-17 PATIENTS WITH HEPATOCELLULAR CARCINOMA HAVE HIGHER RISK AMAP SCORES UP TO 5 YEARS BEFORE DIAGNOSIS}

Benjamin Giles*, Tushar Das, Zeshan Riaz, Joanna Dowman, Andrew Fowell, Richard Aspinall. Portsmouth Hospitals University NHS Trust, Portsmouth, UK

\subsection{6/gutinl-2021-BSG.207}

Introduction Many patients with hepatocellular carcinoma (HCC) are diagnosed too late to be offered potentially curative therapies and there is an unmet need for earlier detection. A recent international study assessed the 'aMAP' score (age, male gender, albumin-bilirubin, platelets) for stratifying patients with chronic liver disease into low $(<0.2 \%)$, medium (0.4-1\%) or high (1.6-4\%) annual risk of developing HCC [Fan R et al, J Hepatol 2020]. However, the data was predominantly from patients with chronic viral hepatitis recruited in tertiary centres, which may have been subject to referral bias. Consequently, the aim of our study was to assess whether aMAP scoring could have identified patients who developed HCC in an unselected UK population including non-viral liver diseases.

Methods A prospectively recorded liver cancer database was used to identify all patients diagnosed with HCC according to EASL-EORTC criteria, over a 10 -year period at a major acute 
hospital serving a population of 675,000 . Clinical data included demographics, laboratory parameters, aetiology and stage of liver disease. The aMAP scores were calculated using blood tests at intervals from 1-5 years prior to diagnosis of HCC to classify patients as low, medium or high risk.

Results We identified 226 patients diagnosed with HCC between 2010 and 2020. Of these, 181 (80\%) were male, median age at diagnosis was 72 years and 215 (95\%) were of white ethnicity. Aetiological factors were alcohol 78 (35\%), non-alcoholic fatty liver disease 68 (30\%), chronic viral hepatitis $54(24 \%)$ and others $20(9 \%)$. There was no prior history of liver disease in 83 (37\%). Only 56 patients (25\%) were in a formal surveillance programme and just 47 (21\%) were of Barcelona Clinic Liver Cancer (BCLC) stage A or lower at diagnosis. However, a medium or high risk aMAP risk score was identifiable prior to HCC diagnosis in $99 \%$ at 1 year, $97 \%$ at 3 years and $96 \%$ at 5 years. A high risk aMAP score alone was found in $76 \%$ at 1 year, $73 \%$ at 3 years and in $67 \%$ at 5 years prior to diagnosis with HCC.

Conclusions Our study examined a 'real world' population with HCC, representative of the broad clinical spectrum of UK liver disease. We confirmed most cases were diagnosed at a non-curative stage and were outside of a formal surveillance programme. Importantly, we have shown the vast majority of patients with HCC had demonstrably higher risk aMAP scores, which were identifiable up to 5 years before diagnosis. Use of aMAP scoring could improve targeting of candidates for HCC screening and our findings would support further, prospective evaluation in people with chronic liver disease in the UK.

\section{PWE-18 METHYLPREDNISOLONE:DIFFERENT OUTCOME IN PREDNISOLONE NON-RESPONSE SEVERE ALCOHOLIC HEPATITIS PATIENTS}

Mohamed Elnagar*, Adam Lawson, Paraskevi Mandalou, Nicholas Taylor, Andrew Austin. Royal Derby Hospital, Derby, UK

\subsection{6/gutjnl-2021-BSG.208}

Introduction Prednisolone treatment of severe alcoholic hepatitis reduces mortality from $18 \%$ to $14 \%$ at d28 (STOPAH) but not at $\mathrm{d} 90$ (30\%). Non-response may be due to steroid-resistance which might be overcome by using intravenous methylprednisolone (MePred).

Methods All patient with mDF $>32$ treated with MePred over a 4 year period were reviewed. Patients were treated with prednisolone $40 \mathrm{mg}$ daily for 7 days. Prednisolone nonresponse was defined as $\mathrm{d} 7$ Lille model $>0.45$ and severe alcoholic hepatitis confirmed by biopsy. Non-responders were given MePred $500 \mathrm{mg}$ daily for 3 days followed by prednisolone 40mg daily for 25 days and prophylactic antibiotics and fluconazole.

Results Seven non-responders were given MePred given after biopsy confirmation of alcoholic hepatitis. At d7 post-MePred, Lille score was $<0.45$ in 5 patients; one died after intracerebral haemorrhage and 4 survived beyond $\mathrm{d} 90$. Lille score remained more than 0.45 in 2 patients; one failed to respond and died at $\mathrm{d} 9$, the other died of liver failure at $\mathrm{d} 28$ despite a fall in bilirubin $\mathrm{d} 7-431$ to $\mathrm{d} 28-297$ (Figure1). No infective complications were reported. Mortality d28 29\% and d90 $42 \%$.
Bilirubin Changes During Treatment

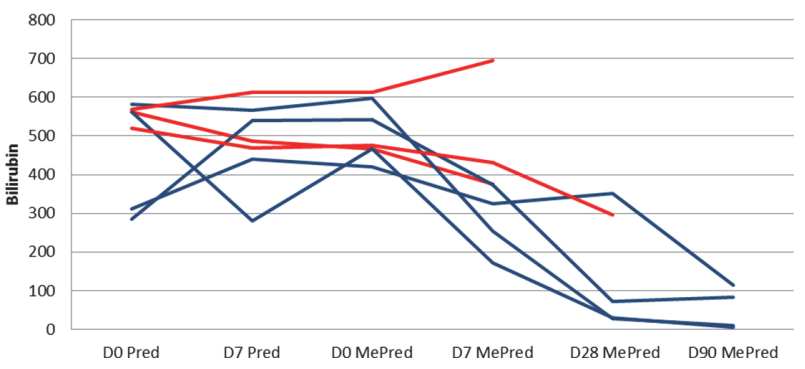

Abstract PWE-18 Figure 1 Bilirubin Changes during Treatment Key: Survivors in blue, Pred: Prednisolone

Conclusions In patients with severe alcoholic hepatitis and prednisolone non-response, methylprednisolone leads to clinical and biochemical response. Observed 6 months survival in our small cohort following MePred was $57 \%$ compared to $25 \%$ predicted by Lille model.

\section{PWE-19 THE ASSOCIATION OF EXERCISE PARTICIPATION WITH CARDIOMETABOLIC HEALTH AND QUALITY OF LIFE IN HEPATITIS C}

\begin{abstract}
1,2,3Kate Hallsworth*, 'Shion Gosrani, 'Sarah Hogg, ${ }^{1}$ Preya J Patel, 'Aaron Wetten, ${ }^{1}$ Rachael Welton, ${ }^{1,2}$ Stuart McPherson, ${ }^{4,5}$ Matthew Campbell. 'Liver Unit, The Newcastle upon Tyne Hospitals NHS Foundation Trust, Newcastle Upon Tyne, UK; ${ }^{2}$ Translational and Clinical Research Institute, Newcastle University, Newcastle upon Tyne, UK; ${ }^{3}$ Newcastle NIHR Biomedical Research Centre, Newcastle Upon Tyne, UK; ${ }^{4}$ Institute of Health Sciences and Wellbeing, University of Sunderland, Sunderland, UK; ${ }^{5}$ Leeds Institute of Cardiovascular and Metabolic Medicine, University of Leeds, Leeds, UK
\end{abstract}

\subsection{6/gutjnl-2021-BSG.209}

Introduction Hepatitis C virus infection (HCV) is associated with an increased risk of cardiovascular disease (CVD) and reduced health-related quality of life (HRQoL). Although physical activity (PA)/exercise has been shown to reduce CVD risk and improve HRQoL in patients with liver disease, there is limited data in HCV. We aimed to explore the association between PA/exercise levels, CVD risk and HRQoL in patients with $\mathrm{HCV}$ and assess individuals' attitudes to PA/exercise.

Methods Cross-sectional observational study recruiting consecutive patients with HCV from viral hepatitis clinics. Data was collected on CVD risk factors, anthropometry, HRQoL, and the exercise benefits and barriers scale (EBBS).

Results 86 patients were recruited (71\% male, 94\% white, age $52 \pm 13$ years); $49 \%$ of the cohort self-reported to be currently active. Although HRQoL was reduced across the cohort, patients that were regularly 'active' reported significantly higher HRQoL scores across SF36v2 domains compared to their inactive counterparts $(\mathrm{p}<0.05)$. Metabolic and cardiovascular characteristics were no different between groups stratified by PA/exercise status $(p>0.05)$. EBBS scores were similar in the 'active' vs 'inactive' groups, however, patients categorised as 'active' scored significantly higher on the psychological outlook and social interaction sub-scales $(p<0.05)$ than those that were 'inactive'. There were significant associations between EBBS scores and HRQoL $(p<0.05)$.

Conclusions

$\mathrm{PA} /$ exercise is associated with increased HRQoL in patients with $\mathrm{HCV}$ irrespective of clinical parameters. Addressing specific motivators/barriers to exercise for patients will be key to 